\title{
Kernos
}

Revue internationale et pluridisciplinaire de religion grecque antique

3| 1990

Varia

\section{Les oracles de Béotie}

\section{Pierre Bonnechere}

Édition électronique

DOI : $10.4000 /$ kernos.970

ISSN : 2034-7871

\section{Éditeur}

Centre international d'étude de la religion grecque antique

\section{Édition imprimée}

Date de publication : 1 janvier 1990

ISSN : 0776-3824

\section{Référence électronique}

Pierre Bonnechere, «Les oracles de Béotie », Kernos [En ligne], 3 | 1990, mis en ligne le 19 avril 2011, consulté le 30 avril 2019. URL : http://journals.openedition.org/kernos/970 ; DOI : 10.4000/kernos.970 


\section{LES ORACLES DE BÉOTIE}

La Béotie a compté dans l'Antiquité, une quinzaine d'oracles qui, relativement ignorés pourtant d'auteurs locaux comme Hésiode et Pindare, lui avaient valu l'épithète de poluphônos ${ }^{1}$. La classification de ceux-ci, sur les plans mantique et chronologique, précise le tableau vieilli d'Auguste Bouché-Leclercq ${ }^{2}$, ainsi que certains aspects de la mentalité béotienne.

\section{Les oracles des Héros : Trophonios, Amphiaraos et Héraclès}

Héros englouti par la Terre, au fond d'une caverne encore inconnue de Lébadée, Trophonios vaticinait à l'intention des pèlerins qui avaient osé braver les affres de la véritable descente aux enfers menant à son repaire. Là, chaque consultant, mué lui-même en prophète du lieu, recevait en personne la réponse oraculaire par des voix et des visions, dans une atmosphère tout empreinte d'oniromancie. Physiquement et psychiquement éprouvés, les fidèles étaient ramenés à l'air libre et remis à leurs proches, après que le clergé se fut enquis de la révélation héroïque ${ }^{3}$.

En dépit des efforts de la propagande delphique pour le subordonner à l'Apollon pythien, Trophonios resta un nourrisson de Déméter, honoré par les Lébadéens en tant qu'un Zeus aux caractères chthoniens et de près lié à la fertilité, earth spirit cavernicole dont l'oracle peut être projeté avec vraisemblance dans la plus haute Antiquité. Son prestige, considérable pour un oracle typiquement local, fermement établi aux débuts du classicisme, se refléta jusque dans le théâtre d'Aristophane et se maintint honorablement jusqu'à Pausanias, lui-même consultant du héros, dont on perd la trace après 250 de notre ère ${ }^{4}$.

Autre héros prisonnier des voraces entrailles de la Terre, sage et preux de l'expédition des Sept, Amphiaraos exerça ses talents prophé-

1 PLUT., Sur la disparition des oracles, 5.

2 Histoire de la divination dans l'Antiquité, III, Paris, 1880, p. 213-225.

3 PaUS., IX, 39, 1-40, 2. Voir G. Dossin, Une consultation de l'oracle de Trophonios à Lébadée, in $M B$, 25 (1921), p. 209-220.

4 Voir Pierre et Marie BONNECHÈRE, Trophonios à Lébadée. Histoire d'un oracle, in $L E C$ (1989), à paraître. 
tiques à Thèbes d'abord, dans un sanctuaire de localisation discutée 5 . Hérodote y rattache une légendaire consultation de Crésus, puis celle de Mys, l'envoyé de Mardonios qui aurait éprouvé les oracles grecs en vue de l'affrontement de Platées ${ }^{6}$. Étant donné l'absence de données archéologiques sûres, la chronologie de l'oracle est sujette à caution. Placé sur un pied d'égalité, dans les affaires de Mys et du roi lydien, avec Trophonios et l'Apollon pythien, le manteion n'avait pu émerger du néant et force est de lui accorder une notoriété déjà archaïque. Disparu des sources après Hérodote, il est probable qu'il se soit éteint dans la capitale béotienne, à l'époque où fut fondé, vers 420 av. J.-C., sur sol vierge, le grand Amphiareion d'Oropos ${ }^{7}$, sujet de moquerie pour Aristophane et où l'on recourait, ainsi qu'à Thèbes, à l'oniromancie ${ }^{8}$. Malgré les disputes incessantes entre Athènes et Thèbes pour la possession de la cité, le sanctuaire, encore qu'en perte d'activité depuis la domination de Rome, demeura prospère dans sa vocation avant tout iatromantique jusqu'au temps de Pausanias, ainsi que l'attestent de très nombreux ex-voto et plusieurs inscriptions datées, pour les dernières, de la première moitié du IIIe siècle ap. J.-C.9.

Héraclès enfin, rarement versé pourtant dans l'art divinatoire, exerçait à Hyettos, au nord du Copaïs, des activités iatromantiques, évoquées par le Périégète et confirmées par la découverte, en 1972, d'une inscription d'époque hellénistique, indiquant la présence d'un

5 A. Keramopoulos (Génikè katastasis épi Pausaniou, in $A D, 3\left[B^{\prime}\right]$ [1971], p. 261266), se basant sur PAUSANIAS (IX, 8, 3), avait identifié le site avec quelques ruines au sud de Thebes, mais S. SYMEONOGLOU (The Topography of Thebes, from the Bronze Age to Modern Times, Princeton, 1985, p. 108; 136) lui préfêre un site encore non fouillé au sud de la Cadmée.

6 HDT., I, 46-49; VIII, 134; voir R. CRAHAY, La littérature oraculaire chez Hérodote, Paris, 1956, p. 291-293.

7 STRABON (IX, 2, 10) parle d'une translation entre les deux sanctuaires, et PaUsanias (IX, 8, 3) ne parle pas d'oracle à Thèbes. Le site proposé par A. KERAMOPOULOS offrait l'avantage d'un abandon contemporain à la fondation oropéenne; voir en dernier lieu P. ROESCH, L'Amphiareion d'Oropos, in Temples et sanctuaires, éd. G. Roux, Paris, 1984, p. 173-184. L'existence du sanctuaire thébain a été niée par A. ScHACHTER (Cults of Boiotia, Londres, I, 1981, p. 21-23) au moyen d'un raisonnement qui ne m'a pas convaincu.

8 Sur les installations mantiques, voir P. RoEsCH, art. cit., p. 178-184; sur la stoa où incubaient les consultants, voir J.J. CoulToN, The Stoa at the Amphiareion. Oropos. in ABSA, 63 (1968), p. 147-183.

9 Par exemple, IG, VII, 303; 2498; voir P. RoEsch, art. cit., p. 175 n. 3. 
manteion ${ }^{10}$. Le héros y était d'ailleurs honoré sous la forme d'un bétyle de magnétite, pierre aux propriétés tenues pour médicales dans le monde antique $^{11}$.

\section{Les oracles d'Apollon}

Seigneur des Apollons prophétiques béotiens, Apollon Hisménios bénéficia avant même son homologue pythique, au sud-est de la Cadmée, d'un temple en dur, détruit vers 700 et bientôt reconstruit ${ }^{12}$. La prospérité archaïque de l'oracle ne peut être mise en doute, et le silence à son propos dans l'évocation des sanctuaires béotiens de l'Hymne homérique à Apollon, œuvre pro-delphique, alors qu'il était en pleine activité, vient $\mathrm{du}$ fait qu'il était un concurrent gênant ${ }^{13}$, "siège des prophètes véridiques" réputé pour la splendeur de ses trépieds sans nombre ${ }^{14}$, où l'on recourait à l'empyromancie ${ }^{15}$. L'érection d'un nouveau temple au IVe siècle est sans doute liée à l'hégémonie thébaine ${ }^{16}$; puis vint le sac de la cité par Alexandre, et si le culte d'Hisménios se poursuivit, on ne peut l'affirmer de son oracle qui ne donne plus signe de vie après le IVe siècle ap. J.-C. ${ }^{17}$.

Thèbes comptait sur la Cadmée un second oracle d'Apollon, cité par le seul Périégète, le Spodion, qui tirait son nom d'un autel de cendres et

10 PAUS., IX, 24, 3; sur l'inscription, en dernier lieu P. RoEsch, in Teiresias (1979), E. 79.05; bonne argumentation de R. ÉTIENNE et D. KNOEPFLER, Hyettos de Béotie et la chronologie des archontes fédéraux entre 250 et 171 av. J.-C., Paris, 1976, p. 176-185. A. SchaCHTER, (op. cit., II, Londres, 1986, p. 2-3) préfère ne pas attribuer l'oracle, attesté par l'inscription, à Héraclès, arguant qu'il pourrait tout autant se rapporter à Asclépios.

11 R. ÉTIENNE et D. KNOEPFLer, op. cit., p. 178-181. L'Héracleion de Thespies est né de l'imagination d'A. BouCHE-LECLERCQ (op. cit., III, p. 310), l'inscription invoquée ne comportant aucune trace d'oracle.

12 Bon résumé des fouilles chez S. SYMEONOGLOU, op. cit., p. 93.

13 Voir J. DeFraDAs, Les thèmes de la propagande delphique, Paris, 1954, p. 55-62 et S. SYMEONOGLOU, op. cit., p. 97.

14 PIND., Pythiques, XI, 5-11.

15 HDT., VIII, 134; PHILOCHORE, 328 F 193 J.; SoPH., Edipe-roi, 21.

16 Voir A. ScHACHTER, op. cit., I, p. 81.

17 On a dit que l'oracle avait cessé ses activités dès avant 335 av. J.-C. (Ibidem, p. 82), ou au contraire qu'il avait gardé un rôle important jusque dans l'Antiquité tardive (S. SYMEONOGLOU, op. cit., p. 133). Il est probable qu'il se soit éteint après la destruction de la ville : PAUSANIAS ne cite plus au sanctuaire hisménien de fonctions mantiques (IX, 10, 2-6), alors qu'il décrit celles du Spodion tout proche (IX, 11, 7). 
qui procédait par clédonomancie ${ }^{18}$. Le site restant inconnu, toute précision chronologique est exclue, si ce n'est celle que l'oracle fonctionnait encore à la fin du Haut-Empire ${ }^{19}$.

Le manteion d'Acraiphia (act. Perdikovrysi) reste sujet de controverse : en dernière analyse, il semble qu'il fut, dès sa fondation vers 740-700 av. J.-C., consacré déjà à Apollon, sous l'égide duquel se serait développé, vers 580 av. J.-C., dans le proche sanctuaire de Castraki, le culte du héros Ptoios. La qualité et le nombre impressionnants de kouroi, trépieds et autres offrandes retrouvés sur le site prouvent assez l'éclat rayonnant du Ptoion à l'époque archaïque, dont les structures architecturales demeurent inconnues ${ }^{20}$. Les installations mises au jour datent du VIe siècle pour les citernes basses; du Ve ou du IVe pour le temple et une grotte artificielle toute proche ${ }^{21}$. Lorsque Mys passe au sanctuaire, alors sous influence thébaine, c'est un prophète qui vaticine, divination inspirée donc, sans que l'on puisse en savoir plus ${ }^{22}$. Après la destruction de Thèbes, le manteion ne fait plus que figure d'oracle officiel du koinon béotien, où avaient repris autorité Orchomène et la Béotie du nord; la ferveur populaire entre-temps avait failli, et le sanctuaire ne dut pas survivre longtemps à la dissolution de la Confédération au IIe siècle av. J.-C. ${ }^{23}$

Célèbre jadis pour avoir annoncé, par la voix de son prophète Échécrate, la victoire sur les Perses, l'oracle d'Apollon Tégyraios a été retrouvé dans un petit site isolé au nord d'Orchomène. Procédant par inspiration, il avait connu son heure de gloire au temps des guerres

18 Paus., IX, 11, 7; la thèse de M. Holleaux (Études d'épigraphie et d'histoire grecques, I, Paris, 1938, p. 195-209), selon laquelle l'Hisménion et le Spodion ne seraient qu'un seul et unique oracle est actuellement insoutenable.

19 Il est hasardeux, sur la base du seul PAUSANIAs, de faire du Spodion le prédécesseur de l'Hisménion, comme le voudrait S. SyMEONoGLoU, op. cit., p. 129-130.

20 Sur le Ptoion archaïque, voir J. Ducat, (Les kouroi du Ptoion, Paris, 1971, spéc. p. 439-450), qui fait justice des thèses, pertinentes pourtant, de P. GuILLON (Les trépieds du Ptoion, 2 t., Paris, 1943, spéc. II, p. 99-174), notamment à propos de la prétendue usurpation d'Apollon. Le résumé des fouilles et de leurs avatars chez J. DuCAT, op. cit., p. 3-40.

21 Plan, datation et bibliographie chez P.W. WALLACE, Strabo's Description of Boiotia. A Commentary, Heidelberg, 1979, p. 137-138.

22 HDT., VIII, 134; le rôle de la grotte dans les procédés divinatoires n'est pas assuré : voir J. DUCAT, op. cit., p. 26 n. 5; 447-448.

23 Voir P. GuILlon, op. cit., II, p. 73-77; 157-165; P. RoEsch, Thespies et la confédération béotienne, Paris, 1965, p. 136-141; A. SCHACHTER, op. cit., I, p. 70. 
médiques ${ }^{24}$, alors que son origine reste aussi énigmatique que sa déchéance, advenue dès le IVe siècle pour certains, mais plus vraisemblablement au cours de la période hellénistique ${ }^{25}$.

Apollon œuvrait encore au flanc du Cithéron, dans son sanctuaire d'Hysia, inachevé ou en ruine au passage de Pausanias, où autrefois l'eau du puits saisissait d'enthousiasme ceux qui en ingéraient ${ }^{26}$. Tradition locale difficile à apprécier, évoquant un passé depuis longtemps révolu. À Eutrésis enfin, le dieu possédait un culte, mais l'archéologie n'a pu exhumer l'oracle renommé que lui attribuaient deux compilateurs byzantins 27 .

Les manteia apolliniens de Thespies, des monts Thourion et Libéthrion ont beaucoup trop peu de consistance pour être envisagés valablement ${ }^{28}$.

\section{Un oracle de Poséidon à Onchestos et d'Ammon à Thèbes ?}

L'Hymne homérique à Apollon (229-238) se fait l'écho d'un rite sans parallèle, suivant lequel, lors de la traversée du domaine de Poséidon à Onchestos, le conducteur devait abandonner à lui-même le cheval attelé. Si l'animal, privé de guide, renversait son véhicule cahotant sur le chemin, le char était déposé sur un des murs du sanctuaire. Pratique

24 PLuT., Pélopidas, 16, 5-7; Sur la disparition des oracles, 5. Sur la localisation du site, et les fouilles entreprises, voir S. LAUFFER, Topographisches Untersuchungen in Kopaisgebiet, in $A D, 26$ (B') (1971), p. 239-241.

25 Plutarque (cité n. 24) laisse entendre qu'il n'y avait pas si longtemps que l'oracle avait disparu. A. SCHACHTER, (op. cit., I, p. 75), à la suite d'A. BouchÉLECLERCQ (op. cit., III, p. 214) incline en faveur d'une disparition vers 375 av. J.-C., sans raison précise.

26 Paus., IX, 2, 1. Un puits quelque peu semblable existait à Potnia, au sud de Thèbes dont l'eau rendait les juments folles (PAUS., IX, 8, 2). Il serait vain d'en induire l'existence d'un oracle.

27 STÉPH. BYz., s.v. Eutrésis; EUST., in HoMèRE, Iliade, II, 502. Voir A. ScHACHTER, op. cit., I, p. 48; certaines figurines pourraient, ainsi que de nombreux vases, avoir un rapport avec l'oracle, mais rien n'est sûr.

28 Thespies : schol. ad ARATOS, 223; voir M.P. NILSSON, GGR, $\mathrm{I}^{3}$, p. 545 n. 8 : «pure déduction étymologique». Libéthrion : PAUS., IX, 34, 4; PIND., fr. 104b SnellMăhler; PLUT., Sur les oracles de la pythie, 29; STRAB., IX, 2, 25; voir A. BouchéLECLERCQ, op. cit., III, p. 223 et M. DelCOURT, L'oracle de Delphes, Paris, 1955, p. 137. Thourion : voir l'argumentation, trop complexe pour être réaliste, d'A. SCHACHTER, A Boiotian Cult Type, in BICS, 14 (1967), p. 6; ID., Cults of Boiotia, I, Londres, 1981, p. 43-44. 
oraculaire, fondée sur une question posée sous forme d'alternative ${ }^{29}$ ? Les interprétations sont légion, mais toutes s'accordent pour conférer à l'usage valeur de test où se manifestait la volonté divine et par conséquent apparenté à un procédé divinatoire au sens large $\mathrm{e}^{30}$.

L'existence d'un manteion d'Ammon à Thèbes a récemment été inférée de la IVe pythique de Pindare, et son introduction jugée contemporaine du poète, au moment où Delphes l'avait emporté sur la Thèbes oraculaire archaïque. Celle-ci aurait essayé de tenir tête à sa rivale par l'importation d'un culte divinatoire ancien et vénéré 31 . L'hypothèse est hasardeuse : on s'explique mal le silence de Pindare, qui fait référence au culte, celui de Pausanias, qui cite le temple du dieu, et surtout le silence de Plutarque, qui introduit son dialogue sur la disparition des oracles par une digression sur l'Ammon de Siwah juste avant d'en venir, sans transition, aux oracles de Béotie ${ }^{32}$.

\section{Les oracles des Nymphes et de Tirésias}

Le pouvoir mantique des nymphes béotiennes apparaît au Sphragidion, antre du Cithéron où les autochtones auraient été frappés de transe prophétique, mais à une époque dont Plutarque n'évoque plus que le lointain souvenir ${ }^{33}$. La nymphe d'une source, Telphousa, au pied du Tilphoussaion, a pu jouir d'un oracle archaïque, concurrent ruiné par Delphes, si l'on peut en croire les déductions tirées de l'Hymne homérique, qui met en scène Apollon disposé à établir en cet endroit son manteion, - mais trompé par la nymphe qui le dévie sur Crisa et son dragon, avant de périr de la main de l'Archer irrité ${ }^{34}$.

Une autre tradition voulait que la source Telphousa ait abrité les derniers instants du devin Tirésias, enterré à proximité et en lequel certains se proposent de voir le prophète du lieu, dont Plutarque préfêre

29 Hypothèse d'A. Bouché-LeCLERCQ, op. cit., I, Paris, 1879, p. 150; II, Paris, 1880, p. 368.

30 État de la question chez A. ScHACHTER, Homeric Hymn to Apollo, lines 231-328 (The Onchestos Episode) : Another Interpretation, in BICS, 23 (1976), p. 102114.

31 S. SYMEONOGLOU, op. cit., p. 125-126.

32 PIND., Pythiques, IV, passim; PAUS., IX, 16, 1; PlUT., Sur la disparition des oracles, 2-5.

33 Aristide, 11, 4; voir aussi PaUs., IX, 3, 9.

34 III, 244-276; 375-387. Voir J. DEFRADAS, op. cit., p. 67-69; O. KERN, Die Religion der Griechen, I, Berlin, 1926, p. 89 n. 1; A. SCHACHTER, A Boiotian Cult Type, in BICS, 14 (1967), p. 5. 
replacer l'oracle à Orchomène ${ }^{35}$, sans qu'il soit possible de préciser davantage.

À Thèbes en revanche, Tirésias disposait d'un observatoire pour le vol des oiseaux, au dire de Sophocle "rendez-vous de tous les présages», que Pausanias avait encore pu voir sur la Cadmée, à proximité du temple d'Ammon et de Tyché ${ }^{36}$. Sans doute faut-il localiser cet oracle un peu particulier dans la partie nord-est de l'enceinte sacrée, dans un site qui a livré une structure en L appropriée à un oinoskopeion, suspecté d'avoir été fondé à l'époque archaïque et témoignant d'une occupation ininterrompue depuis l'âge classique jusqu'à la période romaine ${ }^{37}$.

\section{Interprétations et conclusions}

Le nombre des oracles béotiens ne peut donc être arrêté, mais peuvent en être exclus avec vraisemblance ceux que l'on a voulu accorder à Apollon à Thespies, sur les monts Thourion et Libéthrion, et à Ammon sur la Cadmée thébaine. Les manteia de Tirésias à Orchomène et d'Apollon à Eutrésis sont trop faiblement attestés, tandis que ceux du Sphragidion, d'Hysia et d'Onchestos seront admis moyennant des réserves.

Des treize oracles ainsi retenus, seuls quatre procèdent par les signes, l'Hisménion (empyromancie), le Spodion (clédonomancie), l'oinoskopeion (ornithomancie) et l'hypothétique manteion de Poséidon ${ }^{38}$, tous les autres recourant à l'inspiration, prophétique ou incubatoire : la Béotie n'a donc pas échappé à cette tendance grecque de privilégier la divination par l'enthousiasme à la divination inductive ${ }^{39}$.

En présence d'un tel foisonnement d'oracles, la question du pourquoi vient aussi facilement à l'esprit qu'il est malaisé d'y répondre. Et ce n'est pas fuir vers une réponse simpliste pour autant que d'invoquer la mentalité béotienne en elle-même; par nécessité âpre et solide paysan, attaché à la terre et ses invariables travaux, le Béotien

35 PLUT., Sur la disparition des oracles, 44; voir L. BRIsson, Le mythe de Tirésias. Essai d'analyse structurale, Leyde, 1976, p. 30, n. 12; 67-68; J. FONTENROSE, The Spring Telphusa, in TAPhA, 100 (1969), p. 119-131.

36 SOPH., Antigone, 999-1005; schol. ad EUR., Phéniciennes, 840; PAUs., IX, 16, 1.

37 S. SYMEONOGLOU, op. cit., p. 131-132.

38 Sanctuaire dont le mode de divination se rapproche de la cléromancie mais sans se confondre avec elle.

39 Voir par exemple R. BLOCH, La divination dans l'Antiquité, Paris, 1984, p. 6-7. 
demeure un conservateur acharné, pieux et même superstitieux, pétri d'une religiosité aux nombreuses résurgences archaïques ${ }^{40}$. Si la région ne fut pas la seule en Grèce à connaître une sensibilité spirituelle de ce genre, elle fut cependant la seule à avoir développé et surtout conservé, au moins jusqu'à la fin de l'époque classique, pareille activité oraculaire; l'Arcadie, toute sauvage et opiniâtre qu'elle fût, ne connut jamais que trois oracles, mal attestés de surcroît ${ }^{41}$.

Si l'on excepte l'Amphiareion d'Oropos, attique autant que béotien ${ }^{42}$, la pluralité divinatoire de la contrée est axée sur deux pôles essentiels : les quatre oracles thébains, attestant une longue tradition mantique dans la métropole béotienne, et les six manteia du Copaïs, dont quatre gravitent autour d'une montagne et d'une source, d'un héros et d'une nymphe, d'un prophète mâle et d'eau inspiratrice, ce qui pourrait témoigner d'une organisation cultuelle autochtone qu'Albert Schachter a évoquée sans toutefois parvenir à un niveau probatoire décisif ${ }^{43}$.

L'unité des cités du Copaîs n'était pas seulement religieuse, mais forgée aussi autour d'un lac partiellement asséché ${ }^{44}$ qui en conditionnait la vie économique, dépendant de la pêche et de la culture de terrains en partie conquis sur les eaux. Ces eaux, marécageuses au cœur d'une cuvette surchauffée l'été, devaient se muer en générateur chronique de maux épidémiques, et en particulier du paludisme ${ }^{45}$, face auxquels on aimait s'assurer le secours des dieux. Hyettos est un centre

40 Sur l'esprit béotien, on lira P. GuLlon, La Béotie antique, Paris, 1948, p. 63-77.

41 Oracle d'Aléa (Athéna) à Mantinée (IG, V 2, 262), de Pan sur le Lycée (PoRPH., Sur l'antre des Nymphes, 20; schol. ad THÉocR., Idylles, I, 123) et d'Apollon à Gortyne d'Arcadie (ANT. LIB., Métamorphoses, 25); sur les deux premiers, voir M. JoST, Sanctuaires et cultes d'Arcadie, Paris, 1985, p. 180; 374-378.

42 Lors de sa fondation vers 420, l'Amphiareion aurait donc été athénien : voir P. ROESCH, art. cit., p. 175-176.

43 Art. cit., p. 1-16 : les caractéristiques alléguées, bien sûr réelles, restent malgré tout fort vagues. L'extension de ce cult pattern à l'Hisménion me semble en tous cas excessive.

44 Les travaux de drainage du Copaiis remontent à l'époque minyenne : voir J. KNAUss, B. HeINRICH, H. KALCYK, Die Wasserbauten der Minyer in der Kopais, die älteste Flussregulierung Europas, Munich, 1984.

45 Avec comme conséquence une importante stérilité féminine. C'est précisément Trophonios que Xouthos consulte à propos de la stérilité de son épouse Créuse (EUR., Ion, 300-304; 392-394; 404-409). Bien qu'Hésiode n'en parle pas, il est plus que probable que le paludisme ait trouvé en Béotie, et surtout autour du Copaïs, un refuge sûr depuis les temps préhistoriques : voir les arguments de M. GrMeK, Les maladies à l'aube de la civilisation occidentale, Paris, 1983, p. 397-407 (avec bibliographie antérieure). 
iatromantique, le Trophonion ne manque pas de similitudes avec l'Amphiareion et les Asclépeia 46. Par la force des choses, tous les oracles locaux ont dû remplir des fonctions médicales plus qu'occasionnelles, et leur nombre peut être considéré, en partie du moins, comme la conséquence de cet état de choses.

Les manteia béotiens ne connurent qu'un rayonnement très local, avec de rares ouvertures extra-régionales, restreintes aux contrées limitrophes; deux dédicaces du Ptoion pourraient supposer quelques relations avec l'Athènes des Pisistratides, et l'intense activité sculpturale pré-classique générée par le sanctuaire devait avoir suscité certains rapports avec quelques-unes des Cyclades ${ }^{47}$. L'Amphiareion et l'Hisménion thébains, le Ptoion et le Trophonion devaient être suffisamment crédités pour qu'Hérodote accorde foi à la consultation qu'on y rapportait au carien Mys durant l'invasion perse ${ }^{48}$, mais seul Trophonios sut, semble-t-il, tirer parti de sa renommée, en Béotie comme à l'extérieur : une inscription lébadéenne du milieu du IVe siècle cite le nom de plusieurs pèlerins venus, parfois à plusieurs reprises, consulter le héros depuis les régions avoisinantes ${ }^{49}$. L'oracle bénéficie d'une confiance pan-béotienne avant la bataille de Leuctres ${ }^{50}$, fait graver certaines de ses prescriptions jusque dans le sanctuaire du Ptoion et, à Athènes, est suffisamment connu pour être tourné en ridicule par plusieurs auteurs comiques. Il est vrai que la situation de Lébadée sur la route de Delphes ne devait pas compter pour rien dans la connaissance que les Athéniens pouvaient en avoir 51 .

Les autres oracles ne s'illustrent pas. Au reste, la littérature ancienne ne porta que peu d'intérêt à la mantique béotienne, connue pour l'essentiel grâce à Hérodote et à Pausanias, sans oublier Plutarque, unique chantre indigène de la divination locale: si Pindare avait

46 Voir P. et M. BoNNECHÈRE, art. cit. (à paraître).

47 Voir J. DUCAT, op. cit., p. 445-446.

48 Voir note 6.

49 IG, VII, 3055; voir C. VATIN, Le règlement de Lébadée sur la consultation de l'oracle de Trophonios, in F. SALVIAT et C. VATIN, Inscriptions de Grèce centrale, Paris, 1971, p. 81-94. Voir aussi P. et M. BONNECHËRE, art. cit. (à paraître).

50 Voir P. et M. BONNECHËRE, art. cit. (à paraître).

51 Inscription du Ptoion : IG, VII, 4136 (milieu IIIe s. av. J.-C.). Comédies athéniennes de Cratinos, Céphisodore, Alexis et Ménandre, en plus des sarcasmes d'Aristophane : voir les indications de R. KASSEL et C. AUSTIN (éds), Poetae Comici Graeci, IV, Berlin-New York, 1983, p. 239. Je remercie vivement F. JOUAN pour la remarque concernant la situation de Lébadée. 
consacré quelques vers à l'Hisménion et sans doute au Ptoion et à l'Amphiareion ${ }^{52}$, Hésiode, originaire d'Ascra, toute proche du Tilphoussaion, à une vingtaine de kilomètres du Ptoion, de Thèbes, de Lébadée et de Tégyre, n'a cité aucun des oracles béotiens ${ }^{53}$. De plus, il ne parle dans son œuvre, éminemment paysanne et religieuse, d'aucune occasion de consulter par ce biais la divinité, au moment où sans doute plus que jamais la Béotie méritait l'épithète de poluphônos, chère à Plutarque. Un silence énigmatique qui reste sans réponse.

L'absence de fouilles ou l'insuffisance des rapports généraux interdisent toute affirmation solide sur l'époque de fondation des oracles béotiens, en même temps qu'elles restreignent les conclusions quant à leur rayonnement. À l'Hisménion et au Ptoion, les traces d'occupation datent de la seconde moitié du VIIIe siècle, mais si cellesci correspondent probablement à l'établissement des cultes, on pourrait toujours ne les considérer que comme terminus ante quem. Quant à la genèse des autres instituts mantiques, nous en ignorons tout. Seul l'Hymne homérique à Apollon, composé par un proche des milieux delphiques vers 600 av. J.-C., permet paradoxalement de saisir l'importance de certains d'entre eux, dans l'évocation de l'itinéraire d'Apollon, parti de Délos, en quête du havre idéal à l'implantation de son sanctuaire principal : dans les étapes jalonnant son parcours, Thèbes aux quatre oracles est présentée comme un site vierge encore, Onchestos et le Tilphoussaion font l'objet d'excursus importants : autant de sanctuaires réellement concurrents de Delphes au VIIe siècle, du même coup rabaissés pour n'être pas devenus le site idyllique élu par Apollon 54 .

Au lendemain des guerres médiques, la période dorée de la divination grecque s'estompe peu à peu et la mantique béotienne connaît une double évolution. Les oracles d'Apollon qui, le Spodion excepté, recouraient aux services de prophètes, s'endorment dès les siècles classiques pour se trouver à l'agonie durant la période hellénistique : le Ptoion est rapidement réduit à ne subsister qu'au travers des consultations officielles du koinon ou d'Acraiphia, le Tégyraion ne

52 Hisménion : Péans, IX, 39-45; Pyth., XI, 4-11. Ptoion (?) : Péans, IV, fr. 51a-d Snell-Mähler; Péans, VII, fr. 52g Snell-Mähler. Amphiareion (?) : Pyth., VIII, 5476.

53 Hésiode semble d'autre part ne pas avoir eu avec Delphes les rapports qu'on lui a parfois prêtés : voir J. DEFrADAS, op. cit., p. 45-54.

54 Voir J. DEFRADAS, op. cit., p. 55-62; S. SYMEONOGLOU, op. cit., p. 96-97. 
donne plus signe de vie après la guerre du Péloponnèse, l'Hisménion après 335 av. J.-C., Hysia apparaît comme la réminiscence d'un passé depuis longtemps révolu, et tous se sont éteints avant la naissance de Plutarque ${ }^{55}$. Par contre, les oracles des Héros, liés à l'incubation et à l'oniromancie, survivent à la débâcle des sanctuaires apolliniens et à la transformation de la ferveur religieuse des Grecs hellénistiques pour ne disparaître qu'à la fin du Haut-Empire, ou même après.

La dépopulation dont fut victime la Grèce dès avant l'occupation romaine ${ }^{56}$ n'est pas étrangère au phénomène : pas plus que Delphes en effet ${ }^{57}$, les cités béotiennes n'avaient échappé à la chute démographique, - bien marquée sur les rives du Copaïs aux digues à l'abandon et rompues ${ }^{58}$, mais si les oracles étaient désormais trop abondants pour pouvoir subsister, cela n'explique pas pourquoi les oracles apolliniens furent les premiers touchés. À ce propos, les oracles héroïques ont entretenu des rapports très étroits avec la médecine; et l'évolution de la population dut être étroitement liée aux épidémies de paludisme, récurrentes en Grèce depuis l'âge classique. Peut-être le choix des pèlerins s'est-il naturellement porté vers les manteia spécialisés en la matière. Mais surtout, ces mêmes oracles iatromantiques, procédant par incubation, permettaient aux fidèles un contact direct avec la divinité, sans l'intermédiaire d'un prophète qui représentait un écran mal ressenti et susceptible d'erreur. L'incubation fut de la sorte une des dernières institutions du paganisme à persister ${ }^{59}$, avant de passer à la

55 PluT., Sur la disparition des oracles, 5.

56 Sur la dépopulation en Grèce, voir P. SALMON, Population et dépopulation dans l'empire romain, Bruxelles, 1974, p. 117-120; ID., La population de la Grèce antique, in $B A G B$ (1959), p. 466-476. Aux causes du phénomène données par l'auteur, on ajoutera le paludisme sous sa forme la plus grave : M. GRMEK, op. cit., p. 407.

57 Voir G. Roux, Delphes, son oracle et ses dieux, Paris, 1976, p. 214-215.

58 Comme l'a bien souligné J.M. FossEY, The Cities of the Kopaïs in the Roman Periode, in ANRW, II, 7 (1), Berlin-New York, 1979, p. 549-591. PLUTARQUE luimême présentait la dépopulation comme raison première de la perte des oracles béotiens en citant l'exemple de Tégyre et du Ptoion, où ne passaient plus à son époque que de rares bergers et leurs troupeaux (Sur la disparition des oracles, 8 ).

59 J. BEAUJEU (La religion romaine à l'apogée de l'empire, I, Paris, 1955, p. 301 n. 1) estime ainsi qu'au IIe siècle ap. J.-C. quelque 320 Asclépeia étaient encore en activité. 
postérité chrétienne ${ }^{60}$. Le seul oracle d'Apollon encore en fonction sous l'empire fut d'ailleurs le Spodion, où la clédonomancie à l'honneur faisait percevoir au consultant en personne le message divin ${ }^{61}$.

Pierre BONNECHÈRE

Collège Érasme

Aspirant du FNRS

Place Blaise Pascal, 1

B - 1348 LOUVAIN-LA-NEUVE

60 Voir par exemple Aug., Cité de Dieu, XVIII, 5 et XXII, 8, commenté par A. BOUCHÉ-LECLERCQ, op. cit., I, p. 99-101.

61 En ce qui concerne l'oinoskopeion de Tirésias, sa longévité tient probablement au fait que l'importance accordée à l'ornithomancie soit restée grande dans la vie des Grecs et surtout des Romains. 


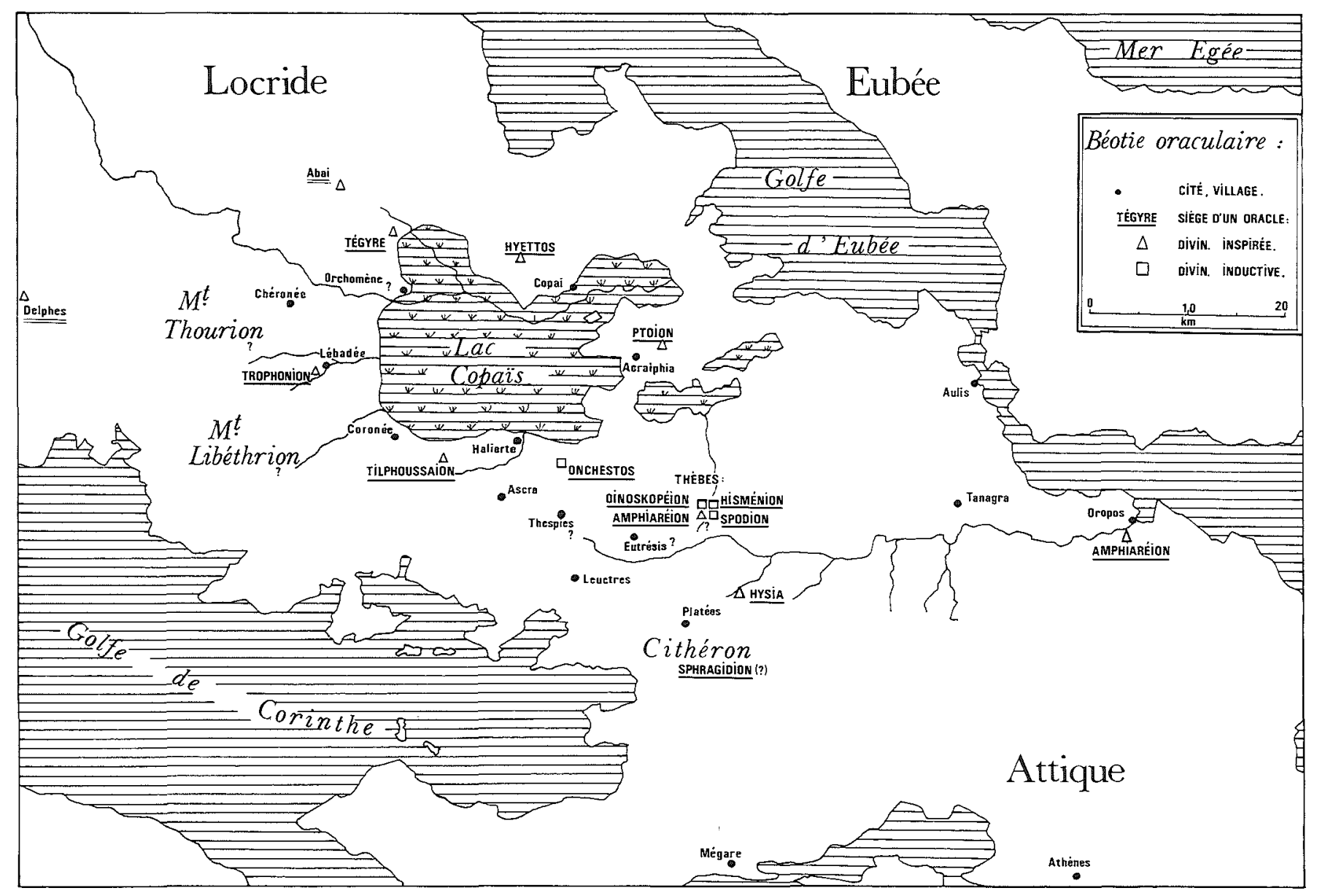

